\title{
A Case of Unerupted Lower Primary Second Molar Associated with Com- pound Odontoma
}

\author{
Unetsubo Teruhisa ${ }^{1}$, Jun Murakami $^{1}$, Miki Hisatomi $^{1},{\text { Yoshinobu } \text { Yanagi }^{2} \text { and Jun-ichi Asaumi }}^{1,2, *}$ \\ ${ }^{I}$ Department of Oral and Maxillofacial Radiology, Field of Tumor Biology, Okayama University Graduate School of \\ Medicine, Dentistry and Pharmaceutical Sciences; ${ }^{2}$ Department of Oral Diagnosis and Dentomaxillofacial Radiology, \\ Okayama University Hospital
}

\begin{abstract}
Odontoma is the most common type of benign odontogenic tumor, and often causes disturbances in the eruption of its associated tooth. Odontomas usually occur in the permanent dentition, and rarely occur solely in the primary dentition. This case report documents a six-year-old-child with a compound odontoma located in the mandible, which caused the impaction of the primary second molar.
\end{abstract}

Keywords: Odontoma, primary tooth, eruption disturbance.

\section{INTRODUCTION}

An odontoma is a mixed tissue benign tumor of odontogenic origin, which exhibits complete dental tissue differentiation. Odontomas constitute about $22 \%$ of all odontogenic tumors of the jaws. The World Health Organization has subdivided odontomas into a compound and complex type based on histologic criteria. Compound odontomas consist of multiple miniature or rudimentary teeth, whereas complex odontomas appear as an amorphous and disorderly pattern of calcified dental tissues. Although odontomas are classified as odontogenic tumors according to the WHO classification, they lack proliferative potentiation. Odontomas are probably a hamartomatous malformation of functional ameloblasts and odontoblasts rather than true neoplasms. The etiology is not clear, although local trauma, infection and genetic factors have been suggested as possible causes [1]. Odontomas are usually detected in school-age children, and the mean age at the time of diagnosis is 14 years [2-4]. The majority of odontomas are asymptomatic and seldom causes swelling, pain, suppuration, bony expansion, and displacement of teeth [2, 5]. These lesions are commonly small, seldom larger than a tooth. Odontomas are generally discovered through routine radiographic examination in dental treatment. An odontoma is essentially a benign lesion, but, often causes disturbances in the eruption of its associated tooth. [6-8]. Odontomas mostly occur in the permanent dentition and are rarely associated with the primary teeth $[5,9,10]$. We have found only six reports of odontomas associated with primary molars in the English literatures [11-16].

In this paper, a rare case of an unerupted lower primary second molar due to the presence of compound odontoma in a young patient is reported and the few similar reports that appear in the bibliography are discussed.

*Address correspondence to this author at the Department of Oral and Maxillofacial Radiology, Field of Tumor Biology, Okayama University Graduate School of Medicine, Dentistry and Pharmaceutical Sciences, 5-1, Shikata-cho, 2-Chome, Okayama-city, Okayama 700-8558, Japan; Tel: +81-86235-6706; Fax: +81-86-235-6709; E-mail: asaumi@ md.okayama-u.ac.jp

\section{CLINICAL REPORT}

A six-year-old-girl was referred to Okayama University Hospital due to the failure of the right lower primary second molar to erupt. Past family and medical histories were unknown. Her general medical history was noncontributory. She was taking no medications. There was no history of trauma to the face or mouth. The lesion was asymptomatic, and there was thus no spontaneous or oppressive pain.

Intraoral examination revealed a mixed dentition period, and all primary teeth except for the right lower primary second molar were present in the mouth. Extraoral examination revealed no abnormal signs in gingiva, buccal tissue and alveolar bone of the right mandible. Periapical and panoramic radiographs revealed that a small single radio-opaque structure was present above the crown of the unerupted lower primary second molar. The mass was surrounded by a narrow radiolucent zone. The root formation of the impacted primary tooth was complete at that time. The successor tooth germs were identified, except for the right lower permanent second premolar. The right lower permanent second premolar appeared to be congenitally missing. Therefore, on the basis of the radiographic evaluations, we diagnosed a compound odontoma interfering with the eruption of the primary tooth (Fig. 1). Surgical removal of the tumor was performed in 2007 with the patient under local anesthesia. After the thin overlying bone was removed, 2 small toothlike structures (sizes: $1 \mathrm{~mm}$ and $5 \mathrm{~mm}$ )-covered with a thick fibrous capsule were revealed. The odontoma was completely removed without disturbing the underlying primary second molar. After surgical removal, the patient was followed up regularly and the impacted primary teeth kept under observation. The diagnosis was confirmed histologically. Histologically, the well-defined tumor mass was composed of 2 toothlike structures exhibiting an irregular arrangement of enamel matrix, dentin cementum, and pulpal tissue. The surface of these structures was partially covered with connective tissue containing an odontogenic epithelium. The histologic diagnosis 
a

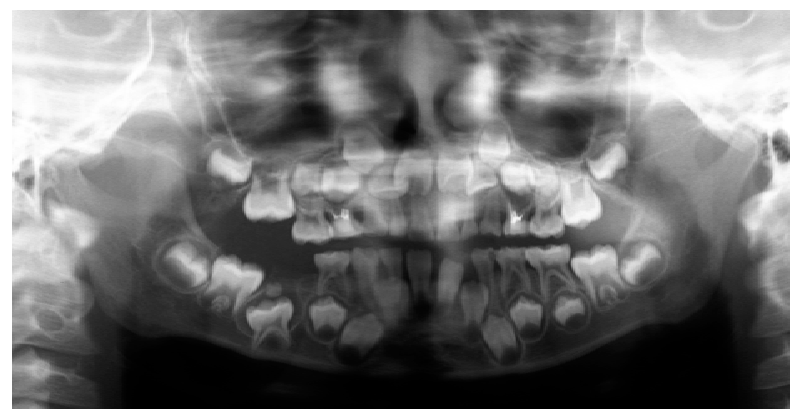

$\mathrm{b}$

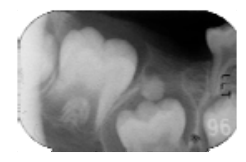

Fig. (1). Radiograph showing compound odontoma and unerupted right lower primary second molar. Radio-opaque mass odontoma was present above the tooth crown of the lower right primary second molar. The tooth germ of the right lower second premolar is absent. (a) Panoramic radiograph. (b) Periapical radiograph before operation.

was a compound odontoma as diagnosed by clinical and radiographic examination. The postoperative period was uneventful.

In this case, there was little chance of spontaneous eruption of the right lower primary second molar because of the complete root formation at the time of diagnosis. The case needs to be referred for orthodontic consultation. An overall evaluation of the occlusion as well as the local problems of impacted second primary molar and congenital missing second premolar should take place.

\section{DISCUSSION}

In clinical setting, dentists often encounter the problem of tooth impaction, which has been defined as a situation where a tooth fails to erupt into a normal functional position by the expected times [11]. Tooth impaction is seen more commonly in permanent dentition and rarely in primary dentition $[17,18]$. For tooth impaction, numerous local etiologic factors have been described. These include odontomas [11, 19, 20], odontogenic tumors [21-23], ankylosis [24], trauma [25], and dentigerous cysts [26]. Among these factors, odontoma is the most common aetiological factor.

In this case report, we presented a rare case of a compound odontoma localized in the mandible, which is associated with the underlying primary second molar. The reason for the impaction of the primary molar was the presence of a compound odontoma. Odontoma is a comparatively common odontogenic tumor, but often causes disturbances in the eruption of teeth [6-8]. In general, odontoma occurs more often in permanent dentition, and rarely associated with primary teeth $[9,10]$. There are very few reports in the literature of odontomas associated with unerupted primary teeth $[5,11,19,20,27-29]$, especially with primary molars [1116]. Various locations of the odontomas associated with primary molars were observed in these reports. One was located between the roots of a right lower second primary mo-

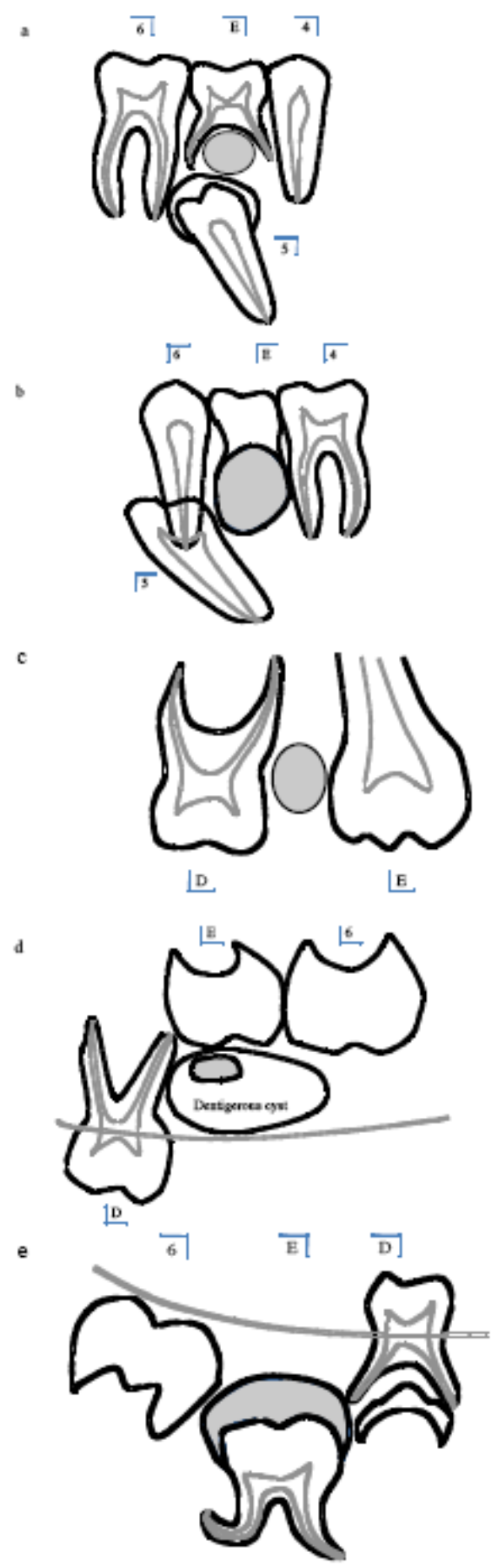

Fig. (2). Location of odontomas (grey painting) associated with primary molars. (a) Compound odontoma located between the roots of the right lower second primary molar (30y, ref.13) (b) Mature complex odontoma seen in a periapical location and in association with the left lower second primary $(25 \mathrm{y}$, ref.14) (c) Odontoma located between the crowns of the upper first and second primary molars (2.6y, ref.15) (d) Unerupted upper second primary molar impacted by an odontoma and a dentigerous cyst (3y, ref.11) (e) Complex odontoma above the tooth crown of the lower right primary second molar (3.7 y, ref.16).

lar (Fig. 2a), one was in a periapical location in association with a left lower second primary (Fig. 2b), one was located 
between the crowns of the upper first and second primary molars (Fig. 2c), one was above the unerupted upper second primary molar (Fig. 2d), and one was above the tooth crown of the lower right primary second molar (Fig. 2e).

The treatment for odontomas in both primary and permanent dentition is their surgical removal. If odontomas are extirpated early without disturbing the underlying tooth germ, the eruption of the impacted teeth can then be expected spontaneously or after orthodontic traction $[2,3,10$, $11,19,28]$. However, underlying impacted teeth are sometimes extracted in association with the removal of odontomas [30]. In this case, the overlying odontoma was surgically removed and the impacted primary tooth kept under observation to monitor its eruption. Periapical and panoramic radiographs revealed that the lower permanent second premolar appeared to be congenitally missing. A full complement of permanent successor tooth germs with the exception of the right lower second premolar was identified. Every effort should be made to preserve lower impacted primary molars. In general, if the root of the impacted tooth is still developing, the tooth may erupt normally; but, once the root apex has closed, the tooth has lost its potential to erupt [31]. Unfortunately, root formation of the impacted molar was complete when we saw the patient. Therefore, it was anticipated that the chance of re-eruption of the impacted right primary molar in this case was low. Interestingly, orthodontic therapy is not usually necessary and is not applied to improve the malocclusion caused by compound odontoma after extirpation of the tumor $[9,32]$. The reason is that most odontomas are very small, and the influence of the tumor on occlusion might be improved without orthodontic therapy. Hisatomi et $a l$. reported that, the impacted tooth tended to erupt regardless of the degree of root formation after extirpation of the odontoma interfering with tooth eruption, although some teeth showed infraversion and/or crowding [16]. However, there have also been some reports about orthodontic therapy which might lead the impacted permanent tooth to a satisfactory postoperative occlusion. Three reports have revealed that impacted primary molars were orthodontically induced to erupt into correct alignment in the dental arch [33-35]. In the present case, considering the slight spontaneous eruption potential of the impacted tooth, we are now planning to apply orthodontic treatment to lead the impacted tooth into its proper position.

In conclusion, the much earlier detection and removal of a compound odontoma associated with an impacted tooth might have contributed to the favorable results for establishing acceptable occlusion.

\section{REFERENCES}

[1] Hitchin AD. The etiology of the calcified composite odontoma. $\mathrm{Br}$ Dent J 1971; 130: 475-82.

[2] Kaugers GE, Miller ME, Abbey LM. Odontomas. Oral Surg 1989; 67: $172-6$

[3] Tandon S, Radhika M. Compound composite odontoma in primary dentition - A case report. J Indian Soc Pedod Prev Dent 1998; 16(4): 111-4.

[4] Kaneko M, Fukuda M, Sano T, Ohnishi T, Hosokawa Y. Microradiographic and microscopic investigation of a rare case of complex odontoma. Oral Surg Oral Med Oral Pathol Oral Radiol Endod 1998; 85: 131-4.

[5] Stajcic ZZ. Odontoma associated with a primary tooth. J Pedodont 1998; 12: 415-20.

[6] Morning P. Impacted teeth in relation to odontomas. Int J Oral Surg 1980; 9: 81-91

[7] Stasinopoulos M. Mixed calcified odontogenic tumors. Brit J Oral Surg 1970; 8: 93-100.

[8] Budnick SD. Compound and complex odontomas. Oral Surg 1976; 42: 501-6.

[9] de Oliveira BH, Campos V, Marcal S. Compound odontoma diagnosis and treatment: three case reports. Pediatr Dent 2001; 23(2): 151-7.

[10] Noonan RG. A compound odontoma associated with a deciduous tooth. Oral Surg Oral Med Oral Pathol 1971; 32(5): 740-2.

[11] Motokawa W, Braham RL, Morris ME, Tanaka M. Surgical exposure and orthodontic alignment of an unerupted primary maxillary second molar impacted by an odontoma and a dentigerous cyst: a case report. Quintessence Int 1990; 21(2): 159-62.

[12] Katz RW. An analysis of compound and complex odontomas. ASDC J Dent Child 1989; 8: 445-9.

[13] Piattelli M, Paolantonio M. Compound odontoma associated with a deciduous molar. Acta Stomatol Belg 1995; 8: 129-30.

[14] Piattelli A, Perfetti G, Carraro A. Complex odontoma as a periapical and interradicular radiopacity in a deciduous molar. J Endod 1996; 8: 561-3.

[15] Long WR, Curbox SC, Cowan JE. Arch-length asymmetry related to an odontoma in a three-year-old. ASDC J Dent Child 1998; 8: 212-3.

[16] Hisatomi M, Asaumi JI, Konouchi H, Honda Y, Wakasa T, Kishi $\mathrm{K}$. A case of complex odontoma associated with an impacted lower deciduous second molar and analysis of the 107 odontomas. Oral Dis 2002; 8(2): 100-5.

[17] Kramer RM, Williams AC. The incidence of impacted teeth. Oral Surg 1970; 29: 237-41.

[18] Bimstein E. Root dilaceration and stunting in two unerupted primary incisors. ASDC J Dent Child 1978; 45(3): 223-5.

[19] Brunetto AR, Turley PK, Brunetto AP, Regattieri LR, Nicolau GV. Impaction of primary maxillary canine by an odontoma: surgical and orthodontic management. Pediatr Dent 1991; 13: 301-2.

[20] Haishima K, Haishima H, Yamada Y, et al. Compound odontomes associated with impacted maxillary primary central incisors: report of two cases. Int J Paediatr Dent 1994; 4: 251-6.

[21] Lysell L, Sund G. Ameloblastic fibroma: report of two cases. Br J Oral Surg 1978; 16: 78-85.

[22] Lukinmaa PL, Heitanen J, Laitinen JM, Malmström M. Mandibular Dentinoma. J Oral Maxillofac Surg 1987; 45: 60-4.

[23] Jameson GD, Bruke PH. Inversion of second deciduous molar and second premolar. Br Dent J 1997; 162: 265-6.

[24] Raghoebar GM, Boering G. An unerupted deciduous molar. Oral Surg Oral Med Oral Pathol 1991; 71: 521-2.

[25] Rothberg MS, Cangiano RJ, Dutante AJ, Maccaro M. Intranasal presentation of an intruded deciduous incisor. Oral Surg Oral Med Oral Pathol 1991; 72: 263.

[26] Boyczuk MP, Berger JR. Identifying a deciduous dentigerous cyst. J Am Dent Assoc 1995; 126: 643-4.

[27] Kilpatrick NM, Hardman PJ, Welbury RR. Dilaceration of a primary tooth. Int J Paediatr Dent 1991; 1: 151-3.

[28] Yassin OM. Delayed eruption of maxillary primary cuspid associated with compound odontoma. J Clin Pediatr Dent 1999; 23(2): 147-9.

[29] Hitchin AD, Dekonor E. Two cases of compound composite odontomes associated with deciduous teeth. Br Dent J 1963; 114: 26-8.

[30] Torreti EF, Carrel R. Compound odontoma in a twelve-year-old girl. ASDC J Dent Child 1983; 50: 376-8.

[31] Kokich VG, Mathews DP. Surgical and orthodontic management of impacted teeth. Dent Clin North Am 1993; 37: 181-204.

[32] Veis A, Tziafas D, Lambrianidis T. A case report of a compound odontoma causing delayed eruption of a central maxillary incisor: clinical and microscopic evaluation. J Endod 2000; 26: 477-9.

[33] Chida R, Chiba K, Saitoh T. A case of an imapacted second deciduous molar caused by anomalies in the position of the tooth 
germ of the succeeding permanent tooth. Shoni Shikagaku Zasshi 1982; 20: 74-80.

[34] Kuba Y, Motokawa W, Ichiki K, Yoshida Y. A case of imapacted mandibular second primary molar following surgical exposure and orthodontic guidance. Shoni Shikagaku Zasshi 1987; 25: 660-70.
[35] Seki N, Ashikaga M, Oka M, Shimooka S. A case of an imapacted maxillary second primary molar following surgical exposure and orthodontic guidance. Shoni Shikagaku Zasshi 1988; 26: 621-30.

(C) Teruhisa et al.; Licensee Bentham Open.

This is an open access article licensed under the terms of the Creative Commons Attribution Non-Commercial License (http://creativecommons.org/licenses/by-nc/3.0/) which permits unrestricted, non-commercial use, distribution and reproduction in any medium, provided the work is properly cited. 